\title{
COVID-19-Pandemie: Belastungen des medizinischen Personals
}

\author{
Ein kurzer aktueller Review
}

COVID-19 Pandemic: Stress Experience of Healthcare Workers

\author{
A Short Current Review
}

\begin{abstract}
Autoren
Jens Bohlken', Friederike Schömig², Matthias R. Lemke ${ }^{3}$, Matthias Pumberger ${ }^{2}$, Steffi G. Riedel-Heller ${ }^{1}$
\end{abstract}

Institute

1 Institut für Sozialmedizin, Arbeitsmedizin und Public Health (ISAP) der Medizinischen Fakultät der Universität Leipzig

2 Centrum für Muskuloskeletale Chirurgie (CMSC), Klinik für Orthopädie, Klinik für Unfall- und Wiederherstellungschirurgie, Universitätsmedizin Berlin, Charité - Campus Berlin Mitte

3 Heinrich Sengelmann Kliniken, Ev. Stiftung Alsterdorf, Hamburg

Schlüsselwörter

COVID-19, Stress, Depression, Angst, medizinisches Personal, Ärzte, Pflege, Review

Keywords

COVID-19, stress, mental health, staff, healthcare worker, physician, nurse, review

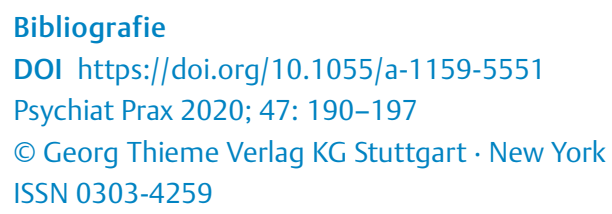

Korrespondenzadresse

Dr. med. Dr. phil. Jens Bohlken, Institut für Sozialmedizin, Arbeitsmedizin und Public Health (ISAP), Universität Leipzig, Medizinische Fakultät, Philipp-Rosenthal-Straße 55, 04103 Leipzig

dr.j.bohlken@gmx.net

\section{ZUSAMMENFASSUNG}

Ziel Darstellung von Studien zur psychischen Belastung von medizinischem Personal unter Bedingungen der COVID-19Pandemie.

Methodik PubMed-gestützte Suche mit den Stichworten COVID 19“, „stress“, „mental health“, „, healthcare worker“, „staff“, „psychiatry“. Eingeschlossen wurden quantitative Studien, (inkl. „Letter to the editor“) zur Belastung des medizinischen Personals im Zeitraum von Januar bis März 2020 .
Ergebnisse Es wurden 14 Studien mit Klinikpersonal aus Infektionsabteilungen, Abteilungen für Fieberkranke, Abteilungen der Inneren Medizin inklusive Intensivstationen sowie der Chirurgie und Psychiatrie identifiziert. Am häufigsten wurden der Patient Health Questionnaire-9 (PHQ-9), die Self-rating-Anxiety Scale (SAS) und die Impact of Event Scale (IES-R) verwendet. Die Stichprobengröße schwankte zwischen 37 und 1257 Personen des überwiegend pflegerischen und ärztlichen Personals. Der Anteil an COVID-19-nahen Tätigkeiten schwankte zwischen 7,5\% und $100 \%$. Es wurde eine erhebliche Belastung durch Stresserleben, depressive und ängstliche Symptome berichtet. Schwere Ausprägungsgrade fanden sich bei 2,2-14,5\% der Befragten. Die Ausprägung der psychischen Symptomatik wurde beeinflusst durch Alter, Geschlecht, Berufsgruppe, Fachrichtung, Art der Tätigkeit und die Nähe zu COVID-19-Patienten. Als Mediatorvariablen wurden das Personalmanagement, die präventive Intervention, die Resilienz und vorhandene soziale Unterstützung angesehen.

Schlussfolgerung Angesichts der Häufigkeit psychischer Symptome bei medizinischem Personal erscheinen begleitende psychiatrisch-psychotherapeutisch informierte Interventionen notwendig, um eine Bewältigung zu unterstützen. Eine schnell einsetzende Forschung ist in diesem Bereich wünschenswert.

\section{ABSTRACT}

Aim Review of studies on the psychological stress of healthcare workers caused by the COVID-19 pandemic.

Methods A literature search of PubMed was performed using the terms "COVID-19", "stress", "mental health", "healthcare worker”, "staff”, "psychiatry”. Quantitative studies (including letters to the editor) published from January to March 2020 were included.

Results 14 studies on healthcare workers in departments of infectiology, internal medicine, and fever wards including intensive care wards as well as surgery and psychiatry, were included. The Patient Health Questionnaire 9 (PHQ9), Self-rating-Anxiety Scale (SAS) and Impact of Event Scale (IES-R) were the most often used test instruments. The sample size ranged between 37 and 1257 participants consisting of mostly nursing and medical personnel. The frac- 
tion of COVID-19-associated activities varied from $7.5 \%$ to $100 \%$. An extensive strain was reported due to stress experience as well as depression and anxiety symptoms. Severe degrees of those symptoms were found in $2.2 \%$ to $14.5 \%$ of all participants. The severity of mental symptoms was influenced by age, gender, occupation, specialization, type of activities performed and proximity to COVID-19 patients. As mediator variables selection of personnel, pre- ventive interventions, resilience, and social support were reported.

Conclusion Considering the frequency of mental symptoms occurring in healthcare workers, accompanying mental health informed interventions to facilitate coping are necessary. Further research in this field is needed.

\section{Einleitung}

Die aktuelle COVID-19-Pandemie belastet seit Ende Dezember 2019 weltweit Gesellschaften unterschiedlichster Art. Die betroffene Bevölkerung leidet direkt unter hohen Infektionszahlen, die mit einer erhöhten Sterblichkeit verbunden sind. Zudem sind die Maßnahmen des Infektionsschutzes wie die sozialen Einschränkungen mit indirekten Gesundheitsfolgen als auch mit erheblichen psychischen Belastungen insbesondere mit Angst und Depression verbunden [1]. Verschiedene aktuelle Kommentare haben auf die Belastung der seelischen Gesundheit in der Bevölkerung hingewiesen [2]. Dabei gewinnt die psychische Gesundheit des medizinischen Personals [3] und die problematische Versorgung in psychiatrischen Kliniken und in gerontopsychiatrischen Einrichtungen [4] eine besondere Bedeutung. Nicht nur im asiatischen, sondern auch im europäischen Raum wurden diese Erfordernisse dargestellt [5, 6].

Die medizinische Versorgung der Bevölkerung ist bedroht, weil die Mitarbeiter des Gesundheitssystems besonderen Belastungen ausgesetzt sind. Für sie besteht u.a. ein erhöhtes Ansteckungs- und Krankheitsrisiko sowie eine erhöhte Sterblichkeit. Drei Reviews mit unterschiedlichen Schwerpunkten fassen die Ergebnisse zu den mentalen Belastungen durch Pandemien und den Erfordernissen des Umgangs mit diesen Herausforderungen zusammen [7-9]. Mit Bezug auf die COVID-19-Pandemie schildert Brooks et al. 2020 Studien zu den psychischen Folgen von Quarantänemaßnahmen aus den Jahren 20032018, oft bei Infektionen wie z. B. SARS oder Ebola [10]. Darunter waren auch Studien, die sich mit den Folgen für das medizinische Personal befassten [11-20]. Es verwundert deshalb nicht, dass das Thema der psychischen Belastung des medizinischen Personals im Rahmen der aktuellen COVID-19-Pandemie aufgegriffen wurde. Erstaunlich sind der Umfang und die Geschwindigkeit, mit der über empirische Ergebnisse bereits jetzt knapp vier Monate nach dem Ausbruch im Dezember 2019 berichtet wurde. Die Studien weisen unterschiedliche Qualität auf. Ein großer Teil wurde nur in der kurzen Form eines „Letter to the editor“ verfasst. Angesichts der drängenden Aktualität soll im Rahmen dieser Arbeit über die Fragestellungen, die Stichproben, die Methoden und die wesentlichen Ergebnisse dieser Studien berichtet werden.

Fragestellung und Ziel dieses narrativen Reviews ist es daher, die verschiedenen Themen zur psychischen Belastung des medizinischen Personals im Rahmen der COVID-19-Pandemie darzustellen und die Ergebnisse der aktuellen Studien aus dem Vier-Monats-Zeitraum (15.12.2019-12.4.2020) zu berichten.

\section{Methoden}

Es erfolgte zum 12.4.2020 eine PubMed-gestützte Suche mit den Stichworten „COVID 19“, „stress“, „mental health“, „, health care worker“, „staff“, „psychiatry“. Eingeschlossen wurden Studien (inkl. „Letter to the editor“) zur Belastung medizinischen Personals im Zeitraum Januar bis März 2020. Berücksichtigt wurden nur quantitative Studien. Einzelfalldarstellungen oder Studien mit einer Fallzahl von unter $\mathrm{n}=20$ wurden ausgeschlossen. Ergänzt wurde ab dem 1.4.2020 die Suche durch tagesaktuelle Beachtung ausgewählter landesspezifischer Fachzeitschriften. Dazu zählten Der Nervenarzt, British Journal of Psychiatry, American Journal of Psychiatry, Psychiatry Research und Acta psychiatrica scandinavia. Die letzte Kontrolle erfolgte am 12.4.2020.

\section{Ergebnisse}

Es wurden insgesamt 14 Studien [21-34] in die Darstellung aufgenommen. Eine Übersicht zu den Studien in alphabetischer Reihenfolge des Erstautors gibt $>$ Tab. 1. Aufgeführt werden Ziel der Untersuchung, Angaben zur Studienpopulation, verwendete Untersuchungsverfahren, Hauptergebnisse und Schlussfolgerungen.

\section{Unterschiedliche Fragestellungen}

Der überwiegende Teil der Studien diente der Erfassung der mit der Belastung durch die COVID-19-Pandemie einhergehenden psychischen Störungen. Dabei wurde Personal in unterschiedlichen medizinischen Einrichtungen untersucht. Dazu zählten in der Regel Abteilungen für Infektionen und Abteilungen für Fieberkranke, Abteilungen der Inneren Medizin inklusive Notfall- und Intensivstationen, aber auch Abteilungen für Chirurgie [34] und Psychiatrie [30]. Eine Studie untersuchte die Belastungen in einem Vergleich zwischen medizinischem Personal und der Bevölkerung [26]. Andere waren aufgrund des komplexeren Studiendesigns in der Lage, Risikoanalysen [24, 25] durchzuführen und Hypothesen zu testen [33].

\section{Stichprobengrößen, Response- und Expositionsraten}

Stichprobengrößen bestanden aus mindestens 37 [21] und maximal 2299 Personen [28]. Der weit überwiegende Anteil bestand aus Pflegepersonal und Ärzten, aber auch aus Personal aus medizinfernen Bereichen wie z. B. technischer Dienst oder Verwaltung. Die Responseraten der Befragungen schwankten zwischen unter $1 \%$ [22] und $100 \%$ [21]. Die Expositionsraten, 


\begin{tabular}{|c|c|c|c|c|c|}
\hline  & 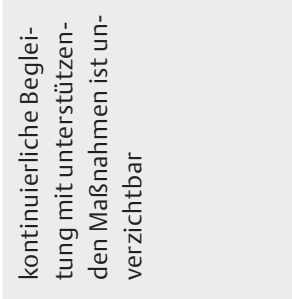 & 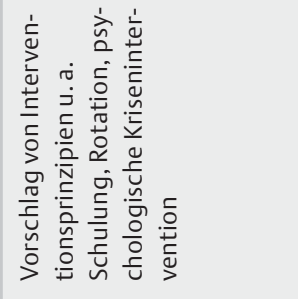 &  &  & 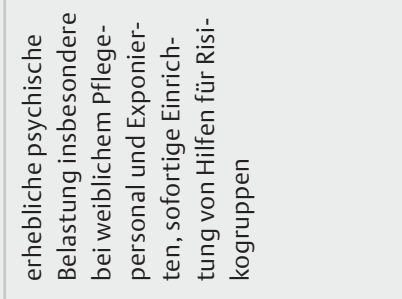 \\
\hline  & 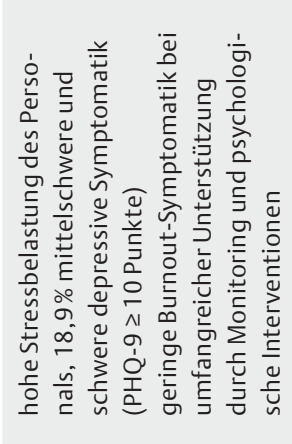 & 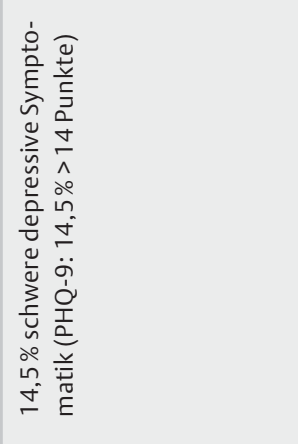 &  & 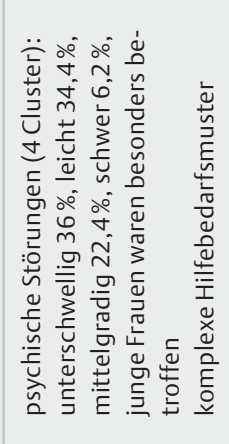 & 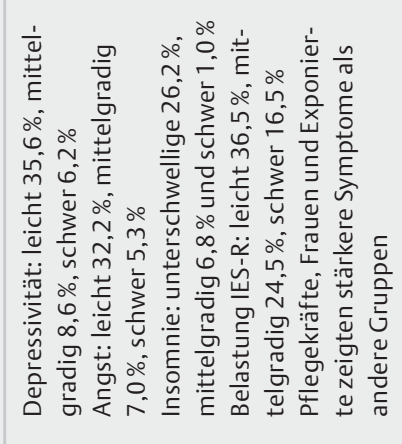 \\
\hline 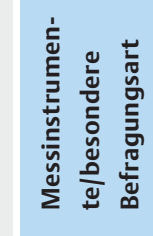 & 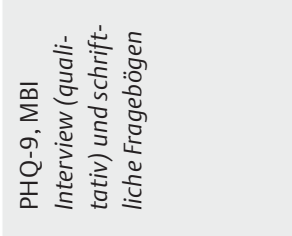 & 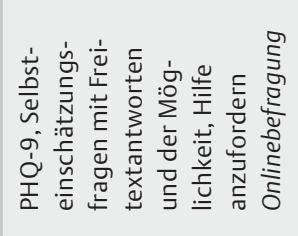 & 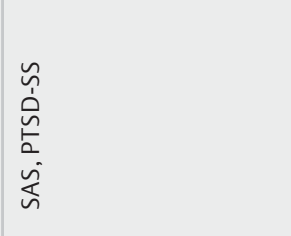 &  &  \\
\hline$\stackrel{\circ}{\check{2}}$ & $\stackrel{\circ}{\circ}$ & $\bar{v}$ & $\begin{array}{l}\text { nn } \\
\tilde{\sigma}\end{array}$ & & $\begin{array}{l}\hat{\infty} \\
\infty^{-}\end{array}$ \\
\hline ف & 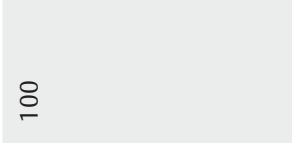 & & 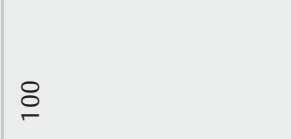 & 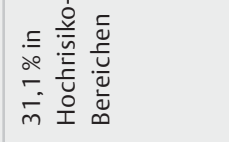 & $\begin{array}{l}\stackrel{n}{\sigma} \\
\stackrel{\sigma}{\sigma}\end{array}$ \\
\hline 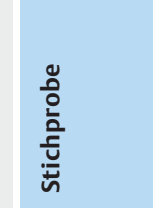 & 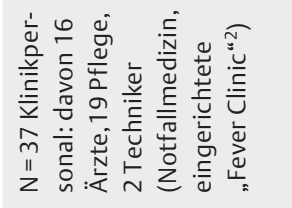 & 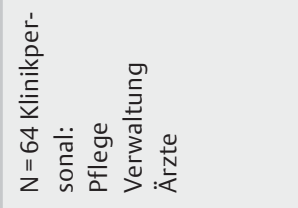 & 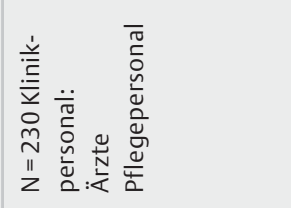 & 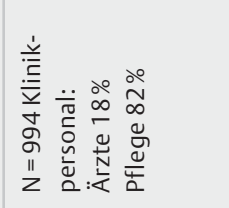 & 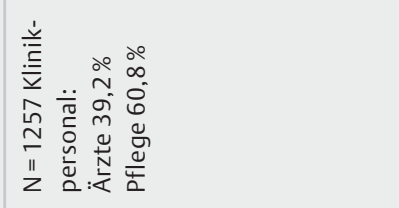 \\
\hline 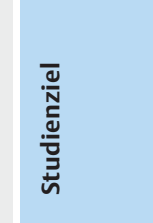 & 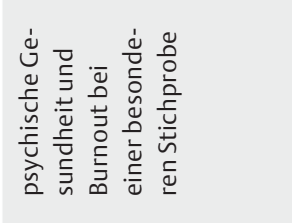 & 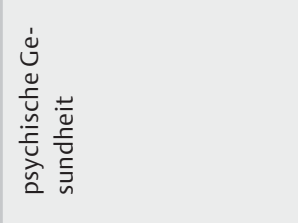 & 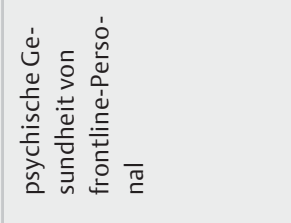 & 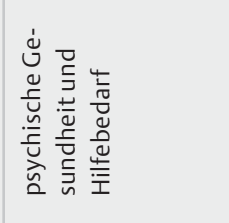 &  \\
\hline  & 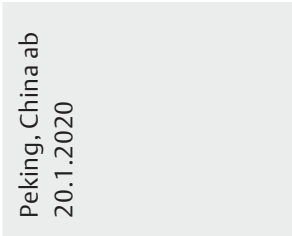 &  & 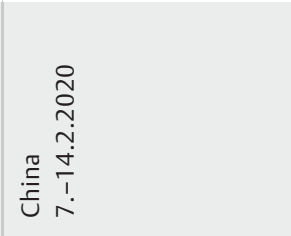 & 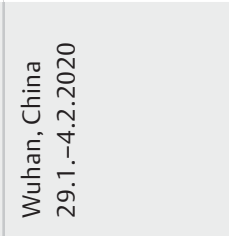 & 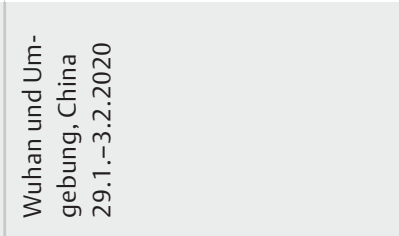 \\
\hline 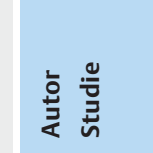 & 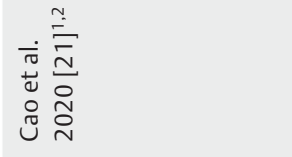 & 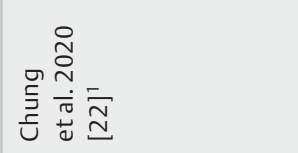 & 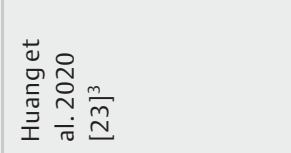 & 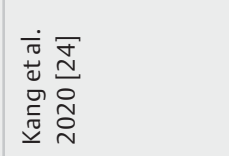 &  \\
\hline
\end{tabular}




\begin{tabular}{|c|c|c|c|c|c|}
\hline 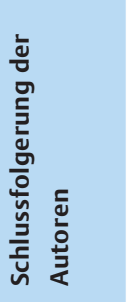 & 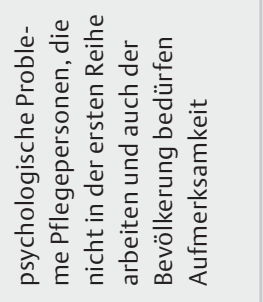 & 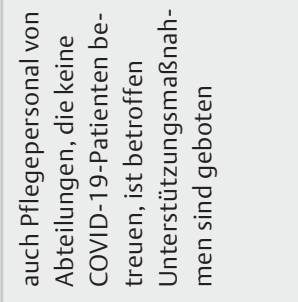 & 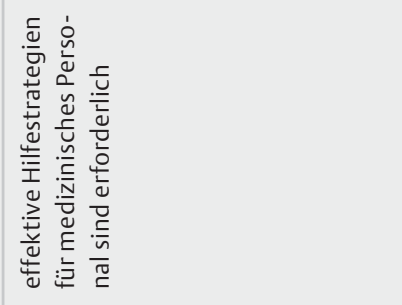 &  & 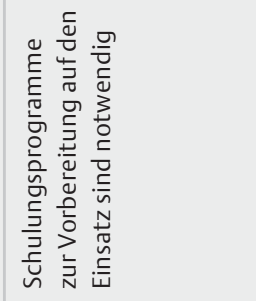 \\
\hline  &  &  & 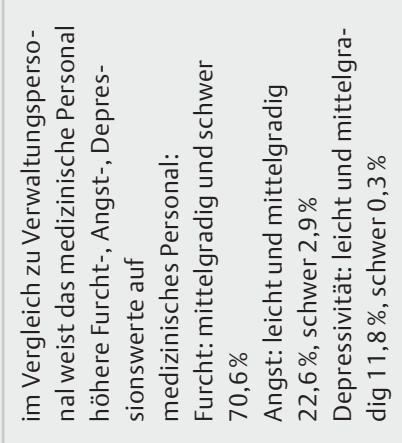 & 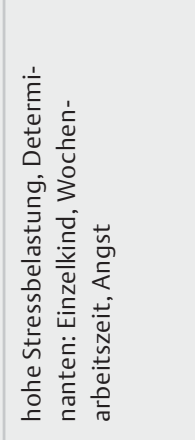 & 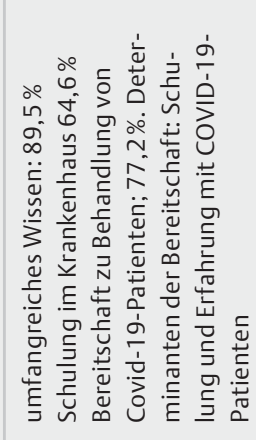 \\
\hline 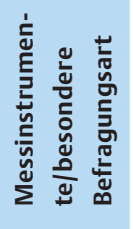 &  & $\begin{array}{l}\tilde{n} \\
\tilde{n} \\
\tilde{\omega}\end{array}$ & 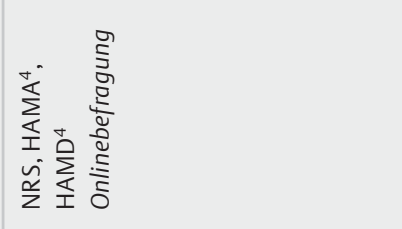 & 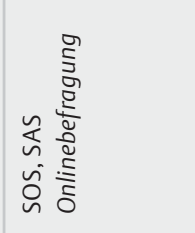 & 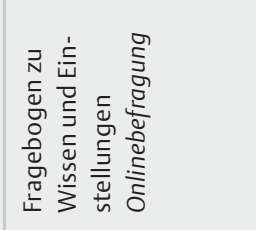 \\
\hline$\stackrel{\circ}{\simeq}$ & & & $\begin{array}{l}\sigma \\
\dot{\sigma}\end{array}$ & $\hat{\omega}$ & \\
\hline 容 & $\begin{array}{l}\mathbf{i} \\
\underset{m}{m}\end{array}$ & $\begin{array}{l}\stackrel{n}{\sim} \\
\stackrel{f}{\sigma}\end{array}$ & $\begin{array}{l}\nabla \\
\stackrel{0}{0}\end{array}$ & $\stackrel{8}{\circ}$ & $\stackrel{\stackrel{n}{r}}{\stackrel{2}{2}}$ \\
\hline 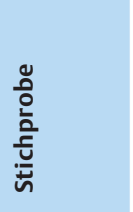 & 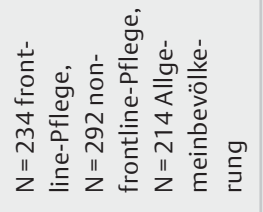 & 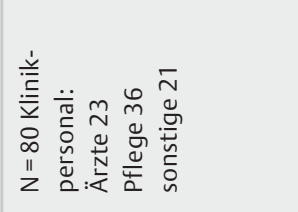 &  & 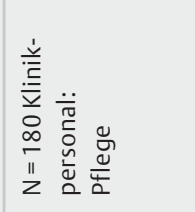 &  \\
\hline  & 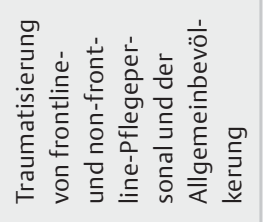 &  &  &  & 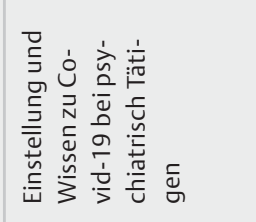 \\
\hline  &  & 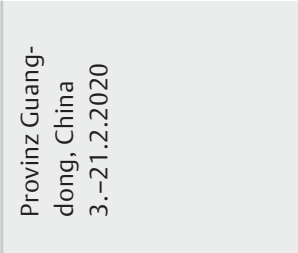 & 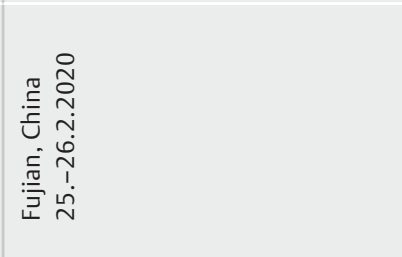 & 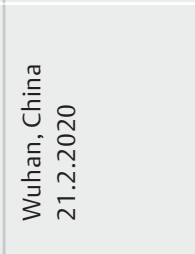 & 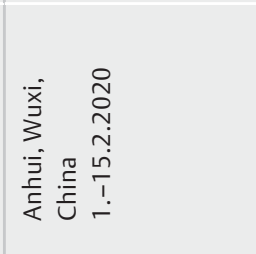 \\
\hline 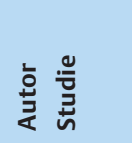 & 离 & 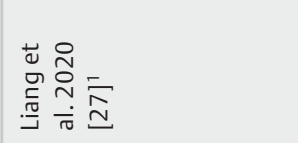 & 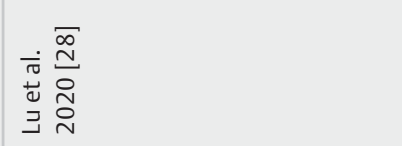 & 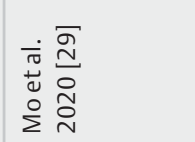 & 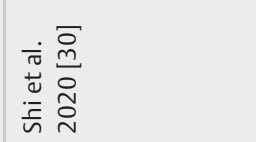 \\
\hline
\end{tabular}




\begin{tabular}{|c|c|c|c|c|c|}
\hline  &  &  & 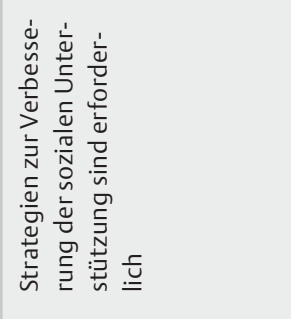 & 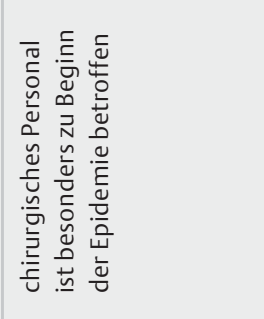 &  \\
\hline 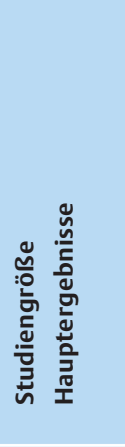 &  & 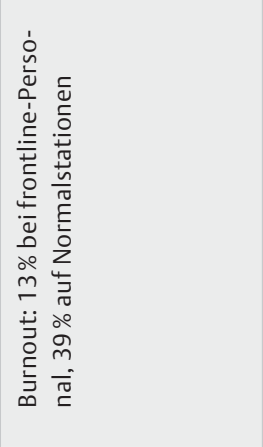 & 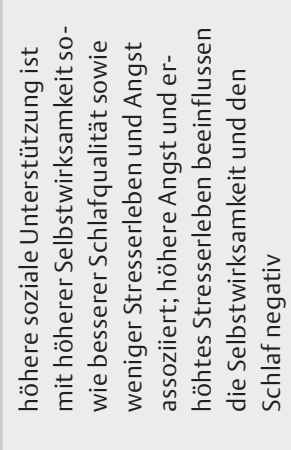 & 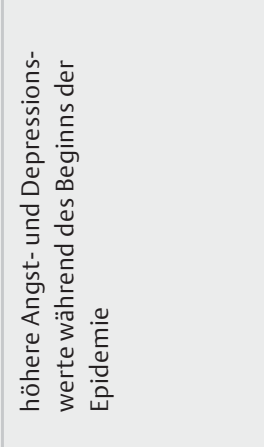 &  \\
\hline 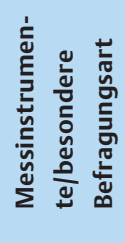 &  & $\overline{\bar{\rho}}$ & 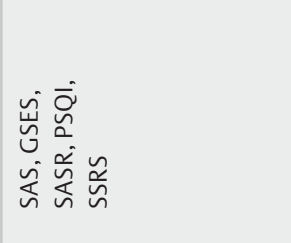 & 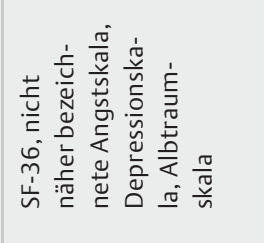 & 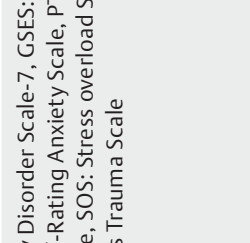 \\
\hline 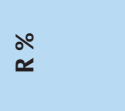 & চ & $\infty$ & $\frac{\infty}{\infty}$ & & 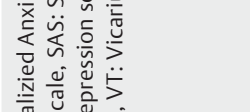 \\
\hline 仓े & $\tilde{0}$ & in & $\stackrel{\circ}{\circ}$ & 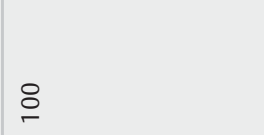 &  \\
\hline 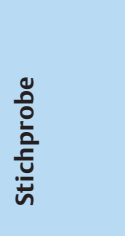 & 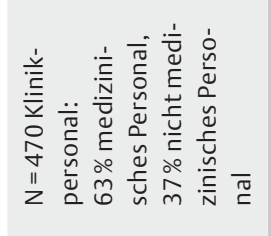 & 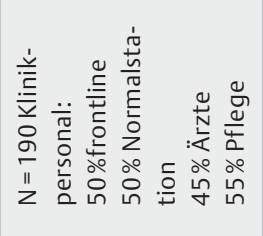 & 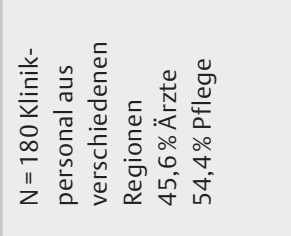 & 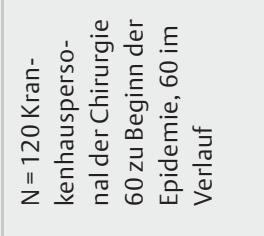 &  \\
\hline 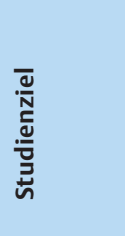 & 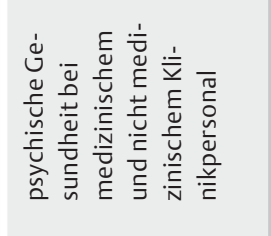 &  & 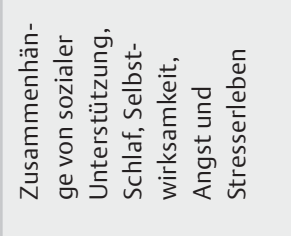 & 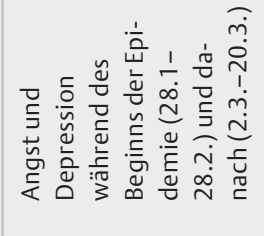 & 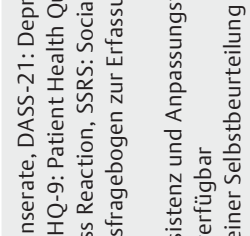 \\
\hline 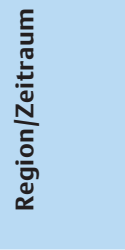 & 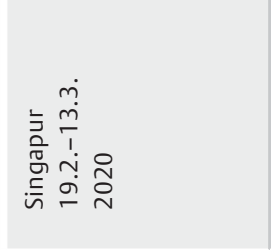 & 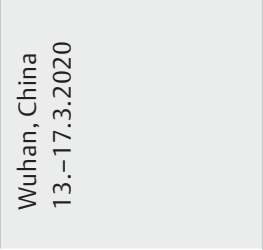 & 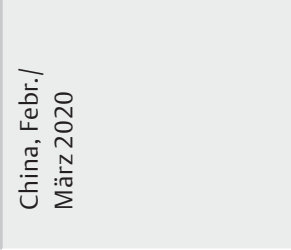 & 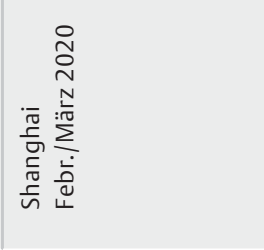 & 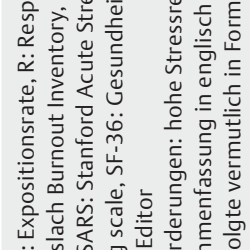 \\
\hline 妾吾 & 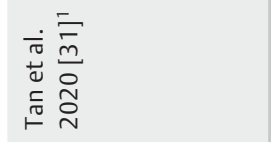 & 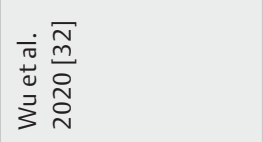 & 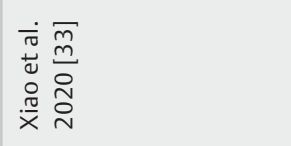 &  & 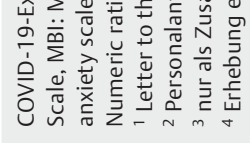 \\
\hline
\end{tabular}


also der Anteil des Klinikpersonals, das in der Nähe von oder im direkten Kontakt mit Patienten mit gesicherter oder möglicher COVID-19-Diagnose arbeitete, schwankte zwischen 7,5\% [30] und $100 \%$ (z. B. [21, 23]).

\section{Untersuchungsmethoden}

Die wesentlichen psychischen Folgen - Stressreaktion, Angst und Depression - wurden durch folgende standardisierte Selbstbeurteilungs-Fragbogenverfahren erhoben. Zur Erfassung der allgemeinen Stressbelastung fanden folgende aus der Stressforschung bekannten Skalen Anwendung: die Impact of Event Scale (IES-R), die Post-Traumatic Stress Disorder Self-rating Scale (PTSD-SS), die Stanford Acute Stress Reaction Scale (SASR) sowie die VT Vicariuos Trauma Scale (VT). Zur Erfassung der Angstsymptomatik gab es folgende Skalen: Generalized Anxiety Disorder GAD-7, Hamilton Anxiety Scale (HAMA), Selfrating-Anxiety Scale (SAS) sowie Self rating Fear Scale (NRS). Die depressive Symptomatik wurde durch folgende Verfahren erfasst: Patient Health Questionnaire-9 (PHQ-9) und Hamilton Depression Scale (HAMD). Bei Schlafstörungen erfolgte die Erhebung mit der Pittsburgh Sleep Quality Scale (PSQI) oder dem Insomnia Severity Index (ISI). Zusätzlich kam das Burnout Inventory (MBI) zum Einsatz. Schließlich erfolgte die Erfassung der Resilienz mit der General-Self-Efficacy-Scale (GSES) und zur Beurteilung der sozialen Unterstützung wurde die Social-Support-Rating-Scale (SSRS) eingesetzt. Am häufigsten wurden der Patient Health Questionnaire-9 (PHQ-9) (vgl. [21, 22, 24, 25]), die Self-rating-Anxiety Scale (SAS) (vgl. [23, 27, 29, 33]) und die Impact of Event Scale (IES-R) (vgl. [24, 25, 31]) verwendet.

Zudem erfolgten teilweise sehr komplexe Erhebungen soziodemografischer Merkmale, die neben Alter, Geschlecht und Berufserfahrung auch detailliert die privaten sozialen und finanziellen Verhältnisse erfassten. Darüber hinaus kamen zum Teil weitere studienspezifische Items oder Fragebögen für die jeweilige Fragestellung zum Einsatz. Erwähnenswert ist, dass in keiner Studie durch die Untersucher ein vollständiger oder strukturierter psychischer Befund erhoben wurde.

\section{Ausprägungsgrade der psychischen Symptomatik}

In $>$ Tab. 1 sind die Belastungen durch Stresserleben, depressive und ängstliche Symptome dargestellt. Betrachtet man nur die am ehesten einer dringenden Intervention oder Behandlung erforderlichen schweren Ausprägungsgrade, dann kann hier von einer Rate von 2,2-14,5\% ausgegangen werden [22-25]. Darüber hinaus ergeben sich in allen Studien bei mehr als einem Drittel des Klinikpersonals Hinweise auf ein leichtes bis moderates Belastungserleben [22-25]. Anders als erwartet, ist offensichtlich auch das Personal betroffen, das nicht unmittelbar mit COVID-19-Erkrankten arbeitet [26, 27].

\section{Risikofaktoren}

Die Ausprägung der psychischen Symptomatik wurde beeinflusst durch Alter, Geschlecht, Fachrichtung, Motivation, Selbstwahrnehmung und vor allem Art der Tätigkeit und Nähe zu COVID-19-Patienten (siehe insbesondere [24, 25]). Als Mediatorvariablen können die Art der Personalrekrutierung, z.B. nach Kriterien der Belastungsfähigkeit und Vorbildung [21], und die angebotenen Interventionen angesehen werden [22, 24, 30]. Bei der Betrachtung der Risikofaktoren ist es von besonderer Bedeutung, auch den Zeitverlauf der Belastung zu berücksichtigen. Nur eine Studie hat diesen Aspekt der Messung des zeitlichen Verlaufs berücksichtigt und dabei den dynamischen Charakter des Belastungserlebens betont [34].

\section{Hinweise auf Interventionen}

Es gibt einige Hinweise auf vorbereitende, kompensierende oder therapeutische Interventionen, die in den untersuchten Kliniken vorgehalten wurden (vgl. [22, 24, 30]). Dazu zählen mediale Vorbereitung [22, 23], schriftliche Informationen [22, 23], spezielle Rekrutierung von Personal [21] für schwierige Aufgaben, gezielte Unterstützung von Stationen mit besonderer Arbeitsbelastung und auch individuelle therapeutische Begleitung.

\section{Diskussion}

In diesem kurzen Review wird über 14 Studien berichtet, die die psychische Belastung von Klinikpersonal innerhalb der ersten Monate nach Ausbruch der Pandemie untersuchten. Das Klinikpersonal war in verschiedensten klinischen Abteilungen beschäftigt. Zu den am häufigsten verwendeten Selbstbeurteilungsverfahren gehörten die Impact of Event Scale (IES-R), der Health Questionnaire-9 (PHQ-9), der Generalized Anxiety Disorder (GAD-7). Die Stichprobengröße schwankte zwischen 37 und 2299 Personen, die meisten davon gehörten zum Pflegepersonal und zu Ärzten. Der Anteil an COVID-19-nahen Tätigkeiten schwankte zwischen 7,5\% und $100 \%$. Es wurde eine erhebliche Belastung durch Stresserleben, depressive und ängstliche Symptome berichtet. Schwere Ausprägungsgrade fanden sich bei 2,2-14,5\% der Befragten. Die Ausprägung der psychischen Symptomatik bei Klinikpersonal wurde durch Alter, Geschlecht, Fachrichtung, Art der Tätigkeit und Nähe zu COVID-19-Patienten beeinflusst. Als Mediatorvariablen können die Art der Personalauswahl und die angebotenen Interventionen angesehen werden.

Zahlreiche, der in diesen Studien untersuchten Themen lassen sich auf deutsche Verhältnisse übertragen. Dazu gehören die Fragestellungen, die verwendeten Untersuchungsinstrumente, die in der Regel auch in validierten deutschen Versionen vorliegen. Interessant wird sein, in welchem Umfang die Belastung von Klinikpersonal in Deutschland mit derjenigen in Asien vergleichbar ist. Auffallend in den asiatischen Studien ist die hohe Beteiligungsrate. Vermutlich ist die sehr hohe Bereitschaft des chinesischen Klinikpersonals, an umfangreichen Befragungsstudien teilzunehmen, in Deutschland weniger ausgeprägt. Zudem ist die Erkrankungswelle in Deutschland nicht mit der gleichen Wucht eingetroffen wie in anderen europäischen Ländern. Obgleich nur eine Studie den dynamischen Charakter des Belastungserlebens betont, erscheint dieser extrem relevant [34]. Möglicherweise wird die vom deutschen Klinikpersonal erlebte Belastung geringer ausfallen und die Zuversicht, die Versorgungsprobleme zu bewältigen, könnte größer sein. Es kann aber auch das Gegenteil der Fall sein. Die Belastung des 
Personals könnte in Deutschland deshalb besonders hoch sein, weil bisher niemand wirklich Erfahrung mit einer solchen Situation hatte und sich auch die Führungskräfte erst an medizinischen und amtlichen Vorgaben orientieren mussten. Darüber hinaus sollte bei deutschen Studien die doppelte Belastung der Mitarbeitenden, nicht nur im professionellen, sondern auch im privaten Bereich (Shutdown, Kontakteinschränkungen, Kinderbetreuung etc.) Berücksichtigung finden. Schließlich sollten diejenigen Personen berücksichtigt werden, die im Bereich der Technik und der Verwaltung für die Bestandserhaltung eines Krankenhausbetriebs oder der kassenärztlichen Verwaltung für den Bestand des medizinischen Versorgungssystems wesentlich sind.

Besonders erstaunlich ist, dass international das medizinische Personal im ambulanten Versorgungssektor bei der Belastungsforschung keine nennenswerte Berücksichtigung findet. Gerade in Deutschland stellt der ambulante Versorgungssektor durch niedergelassene Haus- und Fachärzte eine wichtige Säule des Gesundheitssystems dar und sollte unbedingt in diese Studien einbezogen werden.

Durch den Vorlauf in China und die kanadischen Erfahrungen während der SARS-Epidemie 2003/2004 [14, 15, 19, 20], besteht in Deutschland und auch anderen europäischen Ländern die Möglichkeit, zeitnah Forschungsvorhaben auf den Weg zu bringen. Besonders interessant erscheinen auch Studien, die sich mit Interventionsstrategien für das Pflegepersonal untersuchen. Verdienstvoll sind die deutschen Veröffentlichungen von Petzold und Kollegen zu beurteilen, die sowohl eine breitere medizinische [35] als auch die engere fachärztliche Öffentlichkeit [6] über die besonderen psychischen Belastungen der COVID-19-Pandemie, insbesondere auch des medizinischen Personals informiert haben.

Interessenkonflikt

Jens Bohlken erhielt innerhalb der letzten drei Jahre finanzielle Unterstützung von der Firma Dr. Willmar Schwabe GmbH \& Co. KG und dem Berufsverband Deutscher Nervenärzte (BVDN) für Forschungsprojekte und Vortragstätigkeit. Die anderen Autorinnen/Autoren geben an, dass kein Interessenkonflikt besteht.

Literatur

[1] Wang C, Pan R, Wan X et al. Immediate Psychological Responses and Associated Factors during the Initial Stage of the 2019 Coronavirus Disease (COVID-19) Epidemic among the General Population in China. Int J Environ Res Public Health 2020; 17: 1729

[2] Chew QH, Wei KC, Vasoo S et al. Narrative synthesis of psychological and coping responses towards emerging infectious disease outbreaks in the general population: practical considerations for the COVID-19 pandemic. Singapore Med J 2020. doi:10.11622/smedj.2020046

[3] Chen Q, Liang M, Li Y et al. Mental health care for medical staff in China during the COVID-19 outbreak. Lancet Psychiatry 2020; 7: e15e16

[4] Yao H, Chen JH, Xu YF. Patients with mental health disorders in the COVID-19 epidemic. Lancet Psychiatry 2020; 7: e21. doi:10.1016/ S2215-0366(20)30090-0
[5] Greenberg N, Docherty M, Gnanapragasam S et al. Managing mental health challenges faced by healthcare workers during covid-19 pandemic. BMJ 2020; 368: m1211. doi:10.1136/bmj.m1211

[6] Petzold MB, Plag J, Ströhle A. Umgang mit psychischer Belastung bei Gesundheitsfachkräften im Rahmen der Covid-19-Pandemie. Nervenarzt 2020 [published online ahead of print, 2020 Mar 27]. doi:10.1007/s00115-020-00905-0

[7] Xiang YT, Zhao Y], Liu ZH et al. The COVID-19 outbreak and psychiatric hospitals in China: managing challenges through mental health service reform. Int J Biol Sci 2020; 16: 1741-1744

[8] Koh Y, Hegney DG, Drury V. Comprehensive systematic review of healthcare workers' perceptions of risk and use of coping strategies towards emerging respiratory infectious diseases. Int J Evid Based Healthc 2011; 9: 403-419

[9] Brooks SK, Dunn R, Amlôt R et al. A Systematic, Thematic Review of Social and Occupational Factors Associated With Psychological Outcomes in Healthcare Employees During an Infectious Disease Outbreak. J Occup Environ Med 2018; 60: 248-257

[10] Brooks SK, Webster RK, Smith LE et al. The psychological impact of quarantine and how to reduce it: rapid review of the evidence. Lancet 2020; 395: 912-920

[11] Hawryluck L, Gold WL, Robinson S et al. SARS control and psychological effects of quarantine, Toronto, Canada. Emerg Infect Dis 2004. 10: $1206-1212$

[12] Liu X, Kakade M, Fuller C] et al. Depression after exposure to stressful events: lessons learned from the severe acute respiratory syndrome epidemic. Compr Psychiatry 2012; 53: 15-23

[13] Marjanovic Z, Greenglass ER, Coffey S. The relevance of psychosocial variables and working conditions in predicting nurses' coping strategies during the SARS crisis: an online questionnaire survey. Int J Nurs Stud 2007; 44: 991-998

[14] Maunder RG, Lancee W], Rourke S et al. Factors associated with the psychological impact of severe acute respiratory syndrome on nurses and other hospital workers in Toronto. Psychosom Med 2004; 66: 938-942

[15] Maunder RG, Lancee WJ, Balderson KE et al. Long-term psychological and occupational effects of providing hospital healthcare during SARS outbreak. Emerg Infect Dis 2006; 12: 1924-1932

[16] Wester M, Giesecke J. Ebola and healthcare worker stigma. Scand J Public Health 2019; 47: 99-104

[17] Wu P, Fang Y, Guan Z et al. The psychological impact of the SARS epidemic on hospital employees in China: exposure, risk perception, and altruistic acceptance of risk. Can J Psychiatry 2009; 54: 302-311

[18] Wu P, Liu X, Fang Y et al. Alcohol abuse/dependence symptoms among hospital employees exposed to a SARS outbreak. Alcohol Alcohol 2008; 43: 706-712

[19] Maunder RG, Leszcz M, Savage D et al. Applying the lessons of SARS to pandemic influenza: an evidence-based approach to mitigating the stress experienced by healthcare workers. Can J Public Health 2008; 99: 486-488

[20] Webster P. Canada and COVID-19: learning from SARS. Lancet 2020; 395: 936-937

[21] Cao J, Wei J, Zhu H et al. A Study of Basic Needs and Psychological Wellbeing of Medical Workers in the Fever Clinic of a Tertiary General Hospital in Beijing during the COVID-19 Outbreak. Psychother Psychosom 2020. doi:10.1159/000507453

[22] Chung JPY, Yeung WS. Staff Mental Health Self-Assessment During the COVID-19 Outbreak. East Asian Arch Psychiatry 2020; 30: 34

[23] Huang JZ, Han MF, Luo TD et al. Mental Health Survey of 230 Medical Staff in a Tertiary Infectious Disease Hospital for COVID-19. Zhonghua Lao Dong Wei Sheng Zhi Ye Bing Za Zhi 2020; 38: E001. doi:10.3760/cma.j.cn121094-20200219-00063 
[24] Kang L, Ma S, Chen M et al. Impact on Mental Health and Perceptions of Psychological Care among Medical and Nursing Staff in Wuhan during the 2019 Novel Coronavirus Disease Outbreak: a Cross-sectional Study. Brain Behav Immun 2020. doi:10.1016/j.bbi.2020.03.028

[25] Lai ], Ma S, Wang Y et al. Factors Associated With Mental Health Outcomes Among Health Care Workers Exposed to Coronavirus Disease 2019. JAMA Netw Open 2020; 3: e203976. doi:10.1001/jamanetworkopen.2020.3976

[26] Li Z, Ge J, Yang M et al. Vicarious traumatization in the general public, members, and non-members of medical teams aiding in COVID-19 control. Brain Behav Immun 2020. doi:10.1016/j.bbi.2020.03.007

[27] Liang Y, Chen M, Zheng X et al. Screening for Chinese medical staff mental health by SDS and SAS during the outbreak of COVID-19. J Psychosom Res 2020. doi:10.1016/j.jpsychores.2020.110102

[28] Lu W, Wang H, Lin Y et al. Psychological status of medical workforce during the COVID-19 pandemic: A cross-sectional study. Psychiatry Res 2020; 288: 112936. doi:10.1016/j.psychres.2020.112936

[29] Mo Y, Deng L, Zhang L et al. Work stress among Chinese nurses to support Wuhan for fighting against the COVID-19 epidemic. Journal of Nursing Management 2020. doi:10.1111/jonm. 13014
[30] Shi Y, Wang J, Yang Y et al. Knowledge and attitudes of medical staff in Chinese psychiatric hospitals regarding COVID-19. Brain Behav Immun Health 2020; 4: 100064. doi:10.1016/j.bbih.2020.100064

[31] Tan BYQ, Chew NWS, Lee GKH et al. Psychological Impact of the COVID-19 Pandemic on Health Care Workers in Singapore. Ann Intern Med 2020. doi:10.7326/M20-1083

[32] Wu Y, Wang J, Luo C et al. A comparison of burnout frequency among oncology physicians and nurses working on the front lines and usual wards during the COVID-19 epidemic in Wuhan, China. J Pain Symptom Manage 2020. doi:10.1016/j.jpainsymman.2020.04.008

[33] Xiao H, Zhang Y, Kong D et al. The Effects of Social Support on Sleep Quality of Medical Staff Treating Patients with Coronavirus Disease 2019 (COVID-19) in January and February 2020 in China. Med Sci Monit 2020. doi:10.12659/MSM.923549

[34] Xu J, Xu Q, Wang C et al. Psychological status of surgical staff during the COVID-19 outbreak. Psychiatry Res 2020. doi:10.1016/j.psychres.2020.112955

[35] Petzold MB, Ströhle A, Plag J. COVID-19-Pandemie: Psychische Belastungen können reduziert werden. Dtsch Arztebl 2020; 117: A-648/B552 